\title{
Nanofiber-based $\mathrm{Sm}_{0.5} \mathrm{Sr}_{0.5} \mathrm{Co}_{0.2} \mathrm{Fe}_{0.8} \mathrm{O}_{3-\delta}$ of $\mathrm{N}$-doped graphene modification for bifunctional oxygen electrocatalyst in alkaline medium
}

Yuwei Wang, Ying Yue, Tao Cong, Liquan Fan", Xinyu Su, Xingmei Liu, WeiChao Zhang, Yufeng Li, Xijun Liu

College of Materials Science and Engineering, Heilongjiang Provincial Key Laboratory of Polymeric Composite Materials, Qiqihar University, No.42, Wenhua Street, Qiqihar 161006, PR China *E-mail: Liquan_Fan@163.com

Received: 27 October 2021 / Accepted: 1 December 2021 / Published: 5 January 2022

The development of electrocatalysts with excellent OER and ORR performance is a significant challenge for the commercialization of metal-air batteries. We report $\mathrm{Sm}_{0.5} \mathrm{Sr}_{0.5} \mathrm{Co}_{0.2} \mathrm{Fe}_{0.8} \mathrm{O}_{3-\delta}$ (SSCF28) nanofibers modified by 3D nitrogen-doped graphene (3DNG) composite catalysts with different mass ratios of SSCF28:3DNG. In 0.1 M KOH electrolyte, SSCF28/3DNG with the optimal mass ratio (1:1) demonstrates the best oxygen electrocatalysis performance. The synergistic effect between SSCF and 3DNG benefits the electrocatalytic activities of OER and ORR.

Keywords: $\mathrm{Sm}_{0.5} \mathrm{Sr}_{0.5} \mathrm{Co}_{0.2} \mathrm{Fe}_{0.8} \mathrm{O}_{3-\delta} ; \mathrm{N}$-doped graphene; Nanofibers; OER; ORR

\section{FULL TEXT}

(C) 2022 The Authors. Published by ESG (www.electrochemsci.org). This article is an open access article distributed under the terms and conditions of the Creative Commons Attribution license (http://creativecommons.org/licenses/by/4.0/). 